\title{
INTEGRATING TWO ARTIFICIAL INTELLIGENCE THEORIES IN A MEDICAL DIAGNOSIS APPLICATION
}

\author{
Hadrian Peter ${ }^{1}$, Wayne Goodridge ${ }^{2}$ \\ ${ }^{1}$ University of the West Indies, Barbados; ${ }^{2}$ Dalhousie University, Canada
}

\begin{abstract}
Reasoning Systems (Inference Mechanisms) and Neural Networks are two major areas of Artificial Intelligence (AI). The use of case-based reasoning in Artificial Intelligence systems is well known. Similarly, the AI literature is replete with papers on neural networks. However, there is relatively little research in which the theories of case-based reasoning and neural networks are combined. In this paper we integrate the two theories and show how the resulting model is used in a medical diagnosis application. An implementation of our model provides a valuable prototype for medical experts and medical students alike.
\end{abstract}

Key words: Medical diagnosis, neural networks, case-based reasoning, reasoning system.

\section{INTRODUCTION}

Research in Artificial Intelligence (AI) in medicine has relied on progress in research in knowledge bases and reasoning systems (inference mechanisms). Over the years many medical diagnosis systems - MYCIN, Iliad, DXplain, CADIAG-II, INTERNIST, QMR, and MDDB, to name a few - of which MYCIN [1,2] is arguably the most popular, have been developed. The predominant form of knowledge in MYCIN is represented as a set of rules, and the reasoning system used is backward chaining. Iliad and DXplain [3] both use Bayesian reasoning to calculate probabilities of various diagnoses. CADIAG-II [4] - Computer-Assisted DIAGnosis - is a computer-assisted consultation system to support the differential diagnostic process in internal medicine. CADIAG-II uses fuzzy-based reasoning, however, the underlying knowledge base used is not explicitly described in 
the literature. The knowledge base of INTERNIST, and the strategy used by INTERNIST to address the diagnosis of patients, are described in $[5,6,7,8]$. QMR (Quick Medical Reference) [9, 10, 11], a reengineering of INTERNIST, is an in-depth information resource that helps physicians to diagnose adult disease. However, again, the underlying reasoning and knowledge systems employed in this diagnosis system are not readily available in the literature. Although MDDB [12] uses case-based reasoning, it uses simple lists as its knowledge base. A disadvantage of most of these methods is that, although they exhibit the capability of making differential diagnoses ${ }^{1}$, they do not offer definitive medical consultation ${ }^{2}$. A few earlier attempts at combining the theories of neural networks and case-based reasoning are found in $[13,14,15]$.

In this paper we attempt to correct the shortcomings of the above methods by presenting a new approach to medical diagnosis in which we combine a knowledge base, whose underlying structure is the neural network [16, 17,18], with a Case-Based Reasoning system [19,20,21, 22]. We begin by reviewing case-based reasoning (CBR), we identify problems with such reasoning when used in the medical domain, and provide the motivation for our approach. We then examine neural networks, in particular the mathematical underpinnings of heteroassociative memory neural networks [23], and how they are incorporated in our model. The architecture of our model - the Case-based Memory Network (CBMN) - is introduced in the next section. We then present the medical diagnosis process in our model, followed by the operational model, a short simulation, and a consultation session. The paper ends with a brief evaluation of the model.

\section{MATERIALS AND METHODS}

\subsection{Case-Based Reasoning}

Case-based Reasoning (CBR) [19] is an inference mechanism that has found increasing use in expert systems. It consists of the following four stages: retrieve the most similar case or cases; reuse the retrieved case or cases to solve the problem by analogical reasoning; revise the proposed

\footnotetext{
${ }^{1}$ A differential diagnosis results when 2 or more diagnoses are possible. These diagnoses are prioritized.

2 Definitive medical consultation between the user and the system leads to a concrete diagnosis.
} 
solution; retain the parts of this experience which are likely to be useful for future problem solving.

When CBR is applied to medical diagnosis systems, the following problems are usually identified:

- There is a concentration on reference rather than on diagnosis.

- There is a lack of intelligent dialog. This may result in "missing information" and therefore a decrease of the accuracy of the diagnosis.

- Inability of most similarity algorithms to handle attributes whose values are unknown.

- If the case base contains cases with attributes that take on multiple (rather than just binary) values, then the case base will be quite complex - requiring large numbers of predicates, relations, constraints, and operators [24].

- Updating (revision) of the case base requires complex algorithms and/or highly skilled users.

To overcome these problems, therefore, we developed a variation to the CBR technique called the Case-Based Memory Network (CBMN) model [25]. It was primarily developed to solve medical diagnostic problems and not "pure" classification problems [22]. To simulate the CBMN model we have also designed and implemented an expert system prototype called CaseB-Pro - an interactive system that accepts observed findings, generates appropriate questions, and makes conclusions based on the observed findings.

\subsection{The role of Neural Networks}

The attraction of neural networks in our model is that they have the ability to tolerate noisy inputs and to learn - features which are very desirable in a medical diagnosis system. The CBMN uses a special type of neural network called a heteroassociative neural network [23]. This neural network provides a mechanism for learning, recording what has been learnt, and identifying stored knowledge. The network stores disease patterns associated with cases, and also recalls cases from memory based on the similarity of those cases to the symptoms of the current case. This technique is different from the similarity measure and retrieval techniques such as kdtrees and Case Retrieval Nets (CRNs) [22] employed in CBR. Related classical works in the field of associative memories are found in [26, 27]. 
Let the findings associated with a case be represented by a vector $\mathrm{s}(\mathrm{p})$, where $\mathrm{p}=1,2, \ldots, \mathrm{P}$. Each vector $\mathrm{s}(\mathrm{p})$ is an $\mathrm{n}$-tuple. Let the case associated with findings, $s(\mathrm{p})$, be represented by a vector $\mathrm{t}(\mathrm{p})$. Each $\mathrm{t}(\mathrm{p})$ is an $\mathrm{m}$-tuple.

In our model we store (findings, case) pairs - that is, $\left(\mathbf{s}(\mathbf{p}), \mathrm{t}(\mathbf{p})^{3}\right), \mathrm{p}=$ $1, \ldots . .$, . Here, a "case" is an actual patient, and a "finding" is a symptom, sign, or an investigation. "P" is the maximum number of cases in the database, where

$$
\begin{aligned}
& s(p)=\left(s_{1}(p), \ldots, s_{i}(p), \ldots, s_{n}(p)\right) \text { and } \\
& t(p)=\left(t_{1}(p), \ldots, t_{j}(p), \ldots, t_{m}(p)\right) .
\end{aligned}
$$

We also define a weight matrix $\mathrm{W}_{\mathrm{e}}=\left\{\mathbf{w}_{\mathrm{ij}}\right\}$, where $\mathbf{w}_{\mathrm{ij}}=\sum_{\mathrm{p}} \mathrm{s}_{\mathrm{i}}(\mathrm{p}) \mathrm{t}_{\mathrm{j}}(\mathrm{p})$.

The heteroassociative neural network can be described as a discrete network where the input and output nodes take values from the set $\{-1,0,1\}$. We interpret the values as follows: -1 represents the findings that are absent, 0 represents the unknown findings, and 1 represents the findings that are present. Now $\mathrm{E} \subseteq \Phi$ (observed findings) can be represented as an n-tuple input vector, say $\mathrm{k}$. Vector $\mathrm{k}$ will then be mapped to the $\boldsymbol{\theta}$ domain by the $\mathrm{W}_{\mathbf{e}}$ matrix - findings will be mapped onto cases. That is,

$$
\begin{array}{r}
\text { k. } W_{e} \subseteq \theta \\
\text { or } \quad k \cdot W_{e}=t
\end{array}
$$

where $\mathrm{t} \subseteq \theta$

\section{Example}

If the output layer of the network contains 3 nodes, then some of the following mappings are possible:
k. $W_{\mathrm{e}}=(1,0,0)$
map 1
k. $W_{\mathrm{e}}=(0,1,0)$
map 2
k. $W_{e}=(2,4,2)$
map 3

Whenever new findings are presented to the current case, $\mathrm{k}$ is changed and, when multiplied with the weight matrix $W_{\mathbf{e}}$, the vector $t(p)$ is determined. This value of $t(p)$ is then used to determine a set of actual cases from the case base that matches the observed findings.

\footnotetext{
${ }^{3}$ The term training set is sometimes used to describe the vector $t(p)$.
} 
If a node in vector $t$ has a positive value, then this node represents a case in which the disorder associated with that case matches the current observed findings. For example, in map 1 the disorder associated with case 1 is a possible candidate.

If a mapped vector $t$ contains nodes with varying positive values, then the node with the largest positive value is most likely to be the case that has the most likely associated disorder for the observed findings. For example, if $\mathrm{t}=$ $(3,1,-1)$ then the disorders associated with cases 1 and 2 are likely. However, the disorder associated with case 1 is the more likely candidate.

A disorder, say $\mathrm{k}$, is a part of a definitive diagnosis only if the available findings that will lead to a diagnosis of $\mathrm{k}$ exceed the findings that are known. This serves as the point at which we stop posing questions to the system.

Let current $_{\mathbf{j}}=\Sigma \mathbf{w}_{\mathbf{i j}} \mathbf{x}_{\mathbf{j}}$, unknown input nodes not included and $\quad$ unknown $n_{j}=\sum\left|\mathbf{w}_{\mathrm{ij}}\right|$, only unknown nodes included

If $\mid$ current $_{\mathrm{j}} \mid>$ unknow $_{\mathrm{j}}$, then $\mathrm{k}$ can be a part of the definitive diagnosis.

\subsection{Architecture of the CBMN}

In its simplest form the CBMN structure consists of input information entity (IE) nodes and output (case) nodes. The design goal of the CBMN model is to ensure that a knowledge base, and learning and reasoning mechanisms can be incorporated in the same data structure and be used for diagnostic problem solving. In diagnosing a patient the physician utilizes information from past typical or known exceptional cases that are usually described by a list of symptoms.

To design a medical case base used for diagnostic purposes it is necessary to have two types of cases in the case base [28]:

1. Case Generalizations called prototypes (pure cases) - these are the "classical" (textbook) cases as viewed by the medical expert.

2. General domain cases - these are actual cases.

The features of disorders - in the input layer of the network - are mapped onto case prototypes (in the hidden layer) which represent the "text book" view of disorders in terms of its identifying features. A case representing the output layer of the network - is an instance of a prototype, in the same way that an object is an instance of a class in the object oriented programming paradigm $[29,30]$. The arrows in the diagram denote weighted links from the input nodes to the case nodes, and the calculation, and adjustment, of these weights is known as training the network.

Cases are actual examples of patients and, in the CBMN model, cannot exist without prototypes, which are the physician's representation of 
disorders. That is, every case in the case-base must be associated with one and only one known classical medical case (prototype).

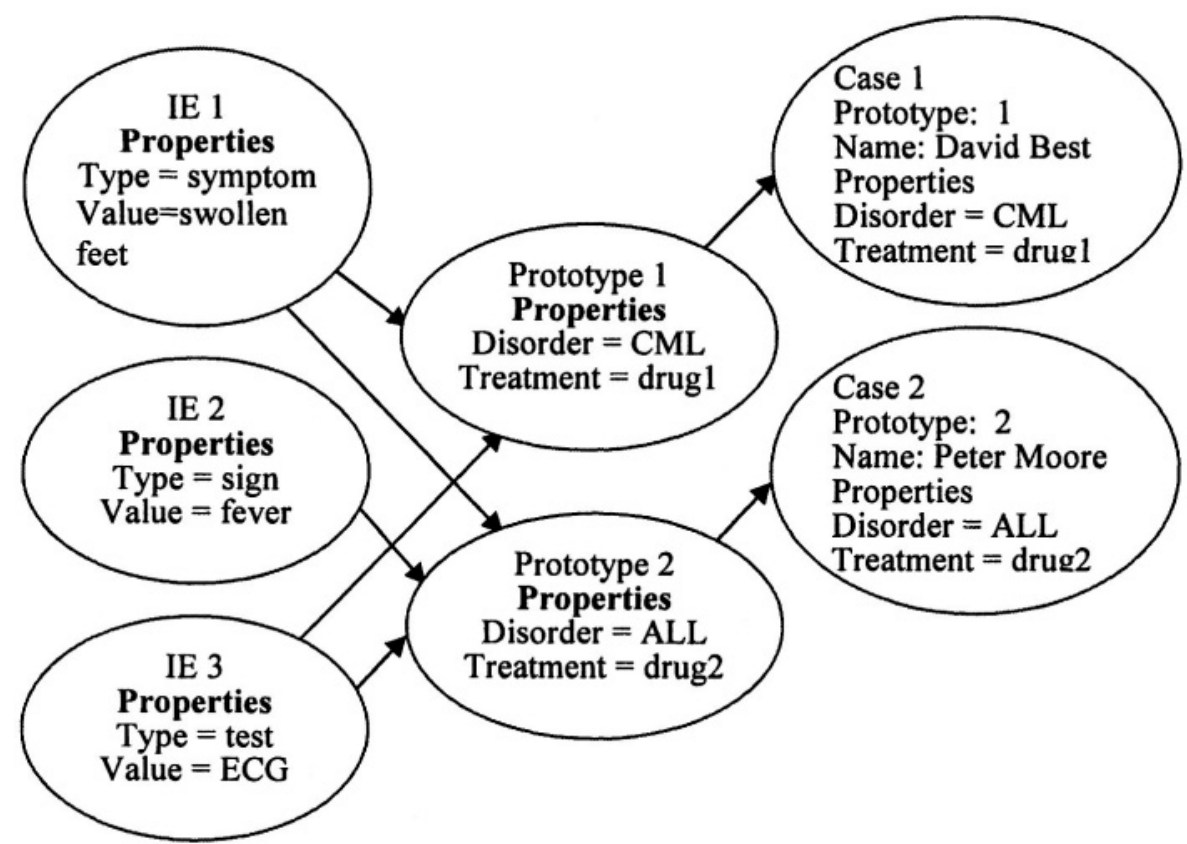

$\underline{\text { Feature IEs }} \quad \underline{\text { Prototypes IEs }} \quad$ Cases IEs

Figure 1. The CBMN Architecture with Prototypes

\subsection{The Medical Diagnostic Process in the CBMN}

A medical consultation consists of the following stages:

- Recording of symptoms and patient history.

- Elicitation / Identification of signs.

- Formulation of notion of diagnosis [31] leading to a hypothesis and differential diagnosis.

- Investigations to narrow down or confirm the diagnosis.

Medical diagnosis depends heavily on known facts about the case in question. The facts are used to form a notion of diagnosis [31], which results in a hypothesis. This hypothesis is strengthened or weakened by discovering more facts about the current case, which in turn invokes a different notion of diagnosis. This process is continued until a definitive diagnosis is found. So, 
again, making a definitive diagnosis is one of the essential differences between CBMN and many extant medical diagnosis systems.

The new approach to the CBMN model includes a certainty factor ${ }^{4}$ [18] and a prevalence factor for each prototype in the case base. A certainty factor is a number ranging from 0 to 10 that represents the physician's impression of the significance of the presence of a feature with respect to the prototype. A prevalence factor is a number ranging from 0 to 10 that expresses the physician's impression that a patient will have the disorder associated with a given prototype. The certainty factor and prevalence factor may or may not be a scientific measurement since it represents only the physician's notion of the disorder.

The presence or absence of features will affect the physician's belief or disbelief in his hypothesis. Hence the concept of the belief factor is used in the CBMN to "balance" a physician's belief and disbelief in a hypothesis.

We now present the algorithm to find the next best question to be asked at a given stage of the medical consultation process. The system cannot reach any definitive conclusions until it has exhausted each stage.

1. Find the set, say S, of prototypes that match the observed findings.

2. Find the prototype, say $\mathrm{k}$, with the highest belief factor in the set $\mathrm{S}$.

3. Use the unknown feature, say $\mathrm{f}$, of prototype $\mathrm{k}$ with the highest certainty factor to generate the next question.

4. If the certainty factor of $\mathrm{f}$ is greater than a question threshold value $\left(\delta_{\mathrm{Q}}\right.$, set by the experimenter), then the system moves to the next consultation stage until the investigation stage is reached.

5. When a diagnostic stage is finished the system lists all the prototypes with a confidence measure greater that a threshold value as candidates for the diagnosis of the presented features.

6. Repeat steps 1-5 above until the investigation stage is completed.

The main objective of this algorithm is to find the optimum diagnostic path that will: (a) Get the correct diagnosis by asking the minimum number of questions and (b) exhaust each diagnostic stage before moving on to the next.

\subsubsection{Simulation}

The simulation of the diagnostic process is implemented in Delphi using a prototype called CaseB-Pro. Its functional specifications can be divided into

\footnotetext{
${ }^{4}$ This model is an example of a truth-functional system for uncertain reasoning.
} 
the following two steps: training the system to identify new cases and using the case base to gain a computer-generated diagnosis.

The two main design goals of the CaseB-Pro prototype - an expert system that combines the theories of neural networks and case-based reasoning - are to implement and test the CBMN model and to develop a computer system that can assist medical students and physicians with the diagnosing of patients. In section 4 we provide an evaluation of the model and an assessment of its "success".

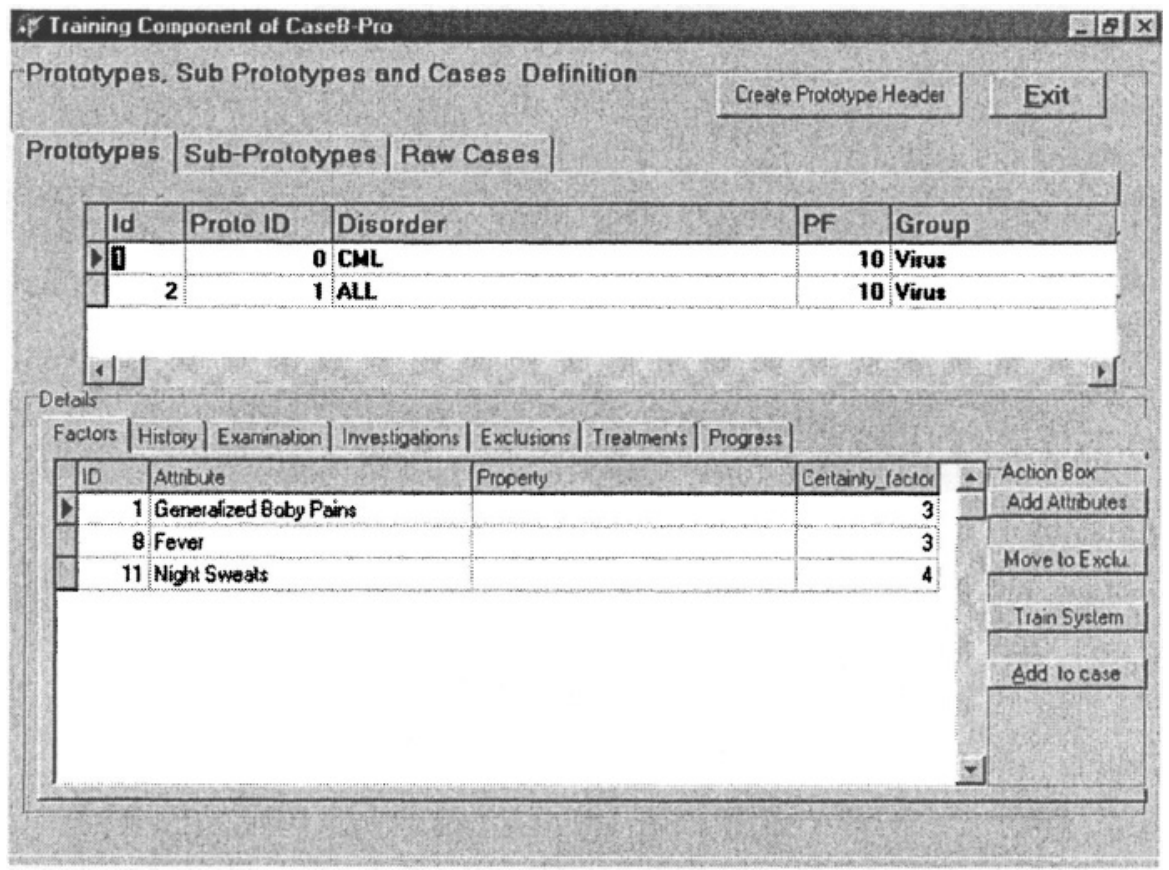

Figure 2. Creating Prototype for CML

The CBMN model uses three types of data structure to represent knowledge. These include: feature information entity data structures, prototype information entity data structures and case information entity data structures.

The training of the model involves adding prototypes to the case base and then, if desired, adding sub-prototypes and actual cases associated with those prototypes. Training also involves the assignment of symptoms, signs, investigations, and exclusions. Training is conducted by using data from classical and actual cases of the disorder in question. The network is trained each time a new case is added to the database, or an existing case is modified. The neural network is used to store and recall cases. Each 
prototype, sub-prototype, and case, of the case base, has in common a node identification number that uniquely identifies the network node in question.

Figure 2 illustrates how the prototype for the Chronic Myeloid Leukaemia (CML) disorder can be added to the system. Other prototypes can be added in a similar manner.

\subsubsection{Interacting with the System}

Figure 3 provides the interface through which users - namely, medical students, physicians, or other medical experts - interact with the CaseB-Pro system. A typical session (consultation) is invoked when a user types the "consult" command. A list of symptoms is then shown where the user can select major presenting symptoms in the case under consideration. The system then allows the user to enter examination results of the case in question. When the examination stage is exhausted the consultation enters into the investigation stage. An example consultation is provided for illustration.

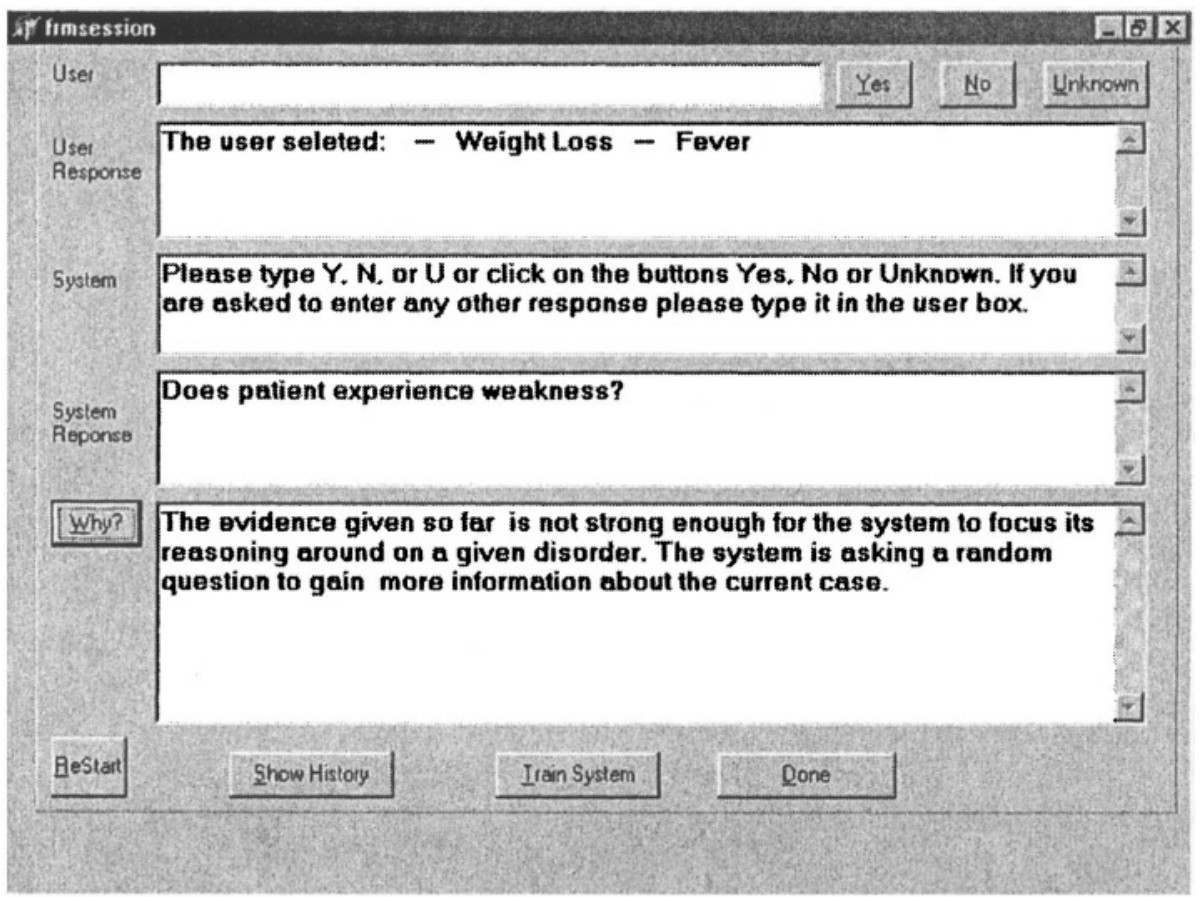

Figure 3. Example of part of consultation session 


\section{RESULTS}

For purposes of testing the system ten cases of Haematological conditions and related disorders were added to the case base. In order to make a preliminary evaluation of CaseB-Pro's diagnostic capabilities, two medical experts who specialize in Haemoncological disorders independently simulated 18 classical Haematological cases within the scope of the system.

Table 1. Results of CaseB-Pro. Generated cases based on medical experts' simulations

\begin{tabular}{|l|l|l|l|l|l|l|}
\hline & $\begin{array}{l}\text { Case } \\
\text { Simulated }\end{array}$ & Stage 1 & Stage 2 & $\begin{array}{l}\text { Opinion- } \\
\text { Stage 1 }\end{array}$ & $\begin{array}{l}\text { Opinion- } \\
\text { Stage 2 }\end{array}$ & $\begin{array}{l}\text { Comments } \\
\text { on DD }\end{array}$ \\
\hline 1 & CML & CML & CML & $\checkmark$ & $\checkmark$ & +++ \\
\hline 2 & MM & MM & MM & $\checkmark$ & $\checkmark$ & + \\
\hline 3 & PRV & PRV & PRV & $\checkmark$ & $\checkmark$ & - \\
\hline 4 & ALL & ALL & ALL & $\checkmark$ & $\checkmark$ & +++ \\
\hline 5 & AA & AA & AA & $\checkmark$ & $\checkmark$ & +++ \\
\hline
\end{tabular}

Key of symbols/abbreviations used in the table:

$$
\begin{aligned}
& \checkmark=\text { Accurate } \\
& \text { DD }=\text { Differential Diagnosis } \\
& \text { +++ = DD Relevant (Related Disorders) } \\
& \text { ++ }=\text { DD Relevant (Unrelated Disorders) } \\
& +\quad \text { = DD Possibly Relevant } \\
& \text { - } \quad=\text { DD Irrelevant } \\
& \text { MM = Multiple Myeloma }
\end{aligned}
$$$$
\text { NHL }=\text { Non Hodgkins Lymphoma }
$$$$
\mathrm{CML}=\text { Chronic Myeloid Leukaemia }
$$$$
\mathrm{MF}=\text { Myelofibrosis }
$$$$
\text { PRV = Polycythaemia Rubra Vera }
$$$$
\mathrm{TB}=\text { Tuberculosis }
$$

Table 1 shows the results of five out of 18 randomly selected simulated interactions. It is, however, important to note that although only 5 of the 18 cases are included in the table, in none of the 18 cases did the medical expert and CaseB-Pro arrive at totally different diagnoses. More specifically, in 9 of the $18(50 \%)$ of the cases the diagnoses were the same at the end of stage 2 (see section 2.4) of the medical consultation. Of the 9 cases in which the diagnoses did not match exactly at the end of stage 2, $7(77.8 \%)$ of them resulted in a match after the differential diagnosis (third) stage. The remaining 2 cases, for which concrete results were not possible at the end of stage 3, produced results in concurrence with the medical expert's diagnoses after further investigations were conducted (in stage 4).

As indicated in section 2.4.1 CaseB-Pro was also used as a teaching tool. To test this feature of CaseB-Pro, two students in the advanced stage of their medical studies were encouraged to interact with the system. Initially the students complained that they felt intimidated by the system and feared that 
the system would expose their lack of knowledge. However, after being persuaded by their medical instructor to use the system as a classroom exercise, the students were noticeably more relaxed. Under the guidance of their medical professor, the students were allowed to conduct an extensive interaction with the system in an effort to diagnose MM, MF, PRV, ALL, and AA (please see the table below). In $80 \%$ of the cases the students were able to arrive at the correct diagnoses largely due, they reported, to the ease with which they were able to follow the "trend of thought" used by CaseBPro.

\section{EVALUATION AND CONCLUSION}

Many medical diagnosis systems have been designed and are currently in use. The originality of our approach, however, is that we have designed and implemented a system that combines case-based reasoning and artificial neural networks. Because of the restriction placed on the length of our paper, we were unable to provide a more detailed comparison with other approaches. Consistent with our goals we were able to (a) implement and test our model, and (b) to develop a computer system that can assist medical students and physicians with the diagnosing of patients. We have been able to develop a prototype, the CaseB-Pro, based on our new approach, whose authenticity medical experts and medical students were able to test.

It may be too early to make strong pronouncements about the success of our model because it was tested on a small domain. Therefore more research using our approach should be conducted using larger domains and different evaluation strategies. Thus far the feedback from persons who have interacted with our prototype has been encouraging, and therefore we are confident that with further development and testing our prototype can evolve into a useful, full-fledged system.

\section{REFERENCES}

1. Buchanan, B.G., Shortliffe, E.H. (eds.): Rule-Based Expert Systems: The MYCIN Experiments of the Stanford Heuristic Programming Project. Addison-Wesley, Reading, Mass. (1984)

2. Shortliffe, B.G. Computer-Based Medical Consultants: MYCIN. Elsevier/North-Holland, Amsterdam (1976)

3. Bouhaddou, O., Sorenson, D.: Iliad V4.4. Journal of Medical Systems, 15(1) (1991) 93-110 
4. Adlassnig, K. -P., Kolarz, G. : Representation and semiautomatic acquisition of medical knowledge in CADIAG-1 and CADIAG-2. Computers and Biomedical Research, 19 (1986) 63-79

5. Miller, R. A., Pople, Jr., H. E., and Myers, J. D. INTERNIST-I, an Experimental ComputerBased Diagnostic Consultant for General Internal Medicine, New England J. Med., 307 (August 1982) 468-476.

6. Pople, Jr., H. E. The Formation of Composite Hypotheses in Diagnostic Problem Solving: An Exercise in Synthetic Reasoning, IJCAI 5 (1977)

7. Pople, Jr., H. E. Heuristic Methods for Imposing Structure on Ill-Structured Problems: The Structuring of Medical Diagnostics, in P. Szolovits (ed.), Artificial Intelligence in Medicine, Boulder, CO (1982) 119-185

8. Masserie, F. E., Miller, R.A., and Myers, J.D. INTERNIST-I properties: Representing common sense and good medical practice in a computerized medical knowledge base. Computers and Biomedical Research, 18 (1985) 458-479

9. Arene, I., et al : Evaluation of quick medical reference (QMR) as a teaching tool. MD Comput., 15 (1998) 323-326

10. Aliferis, C.F., et al: A temporal analysis of QMR. J. Am. Med. Inform. Assoc., 3 (1996) 79-91

11. Miller, R.A., Masarie, F.E.,Jr. : Use of the Quick Medical Reference (QMR) program as a tool for medical education, Methods Inf. Med., 28 (1989) 340-345

12.Gierl, L., Stengel-Rutkowski, S. Integrating Consultation and Semi-automatic Knowledge Acquisition in a Prototype-based Architecture: Experiences with Dysmorphic Syndromes. Artificial Intelligence in Med., 6 (1994) 29-49

13. Myllymaki, P., Tirri, H. Bayesian case-based reasoning with neural networks. In Proceedings of the IEEE International Conference on Neural Networks, San Francisco, CA, 1 (March 1993) 422-427

14. Honkela, T. Self-Organizing Maps and Case-based Reasoning. [Electronic Version] retrieved April, 2004 from http://www.mlab.uiah.fi/networks99/honkela.html

15. Agre, G. Koprinska, I. Case-based Refinement of Knowledge-Based Neural Networks. In: Proceedings of International Conference Intelligent Systems: A Semiotic Perspective, Gaithersburg, MD (Oct. 20-23, 1996)

16. Gallant, S.I.: Neural Network Learning and Expert Systems. MIT, Mass. (1993)

17. Goodridge, W. : The Case-Based Memory Network Model and its Use in Medical Expert Systems: MPhil Thesis, University of the West Indies, Barbados (2000)

18. Russell, S.J., Norvig, P. : Artificial Intelligence : A Modern Approach, $2^{\text {nd }}$ ed. Pearson Education, Inc., New Jersey (2003)

19. Aamodt, A., Plaza, P. Case-Based Reasoning: Foundations Issues, Methodological Variations, and Systems Approaches. AI Communications, 7(1) (1994) 39-59

20. Kolodner, J.L. Case-Based Reasoning. Morgan Kaufmann, New York (1993)

21. Koton, P. Evaluating Case-Based Problem Solving. In: Hammond, K.J. (Ed.). Proceedings of the DARPA Workshop on Case-Based Reasoning, Morgan Kaufmann, CA (1989) 26017022. Lenz, M., et al, (eds.). Case-Based Reasoning Technology: from foundations to 
application. In: Lecture Notes in Computer Science, Vol. 1400. Springer-Verlag, New York (1998)

23. Fausett, L. Fundamentals of Neural Networks. Architectures, Algorithms, and Applications. Prentice-Hall International Editions, New Jersey (1994

24. Bergmann, R., Wilke, W. Case-Based Reasoning: Foundations Issues, Methodological Variations, and Systems Approaches. AI Communications, 3 (1995) 53-118

25. Goodridge, W., Peter, H., Abayomi, A. The Case-Based Neural Network Model and Its Use in Medical Expert Systems. In: Lecture Notes in Artificial Intelligence, Vol.1620. Springer-Verlag, Denmark (1999)

26. Anderson, J. A. A simple neural network generating an interactive memory. Mathematical Biosciences, 14 (1972) 197-220.

27. Kohonen, T. Correlation matrix memories. IEEE Transactions on Computers, 21 (1972) 353--359

28. Rainer, S., Pollwein, B., Gierl, L. Experiences with Case-Based Reasoning Methods and Prototypes for Medical Knowledge-Based Systems. In: Lecture Notes in Artificial Intelligence, Vol. 1620. Springer-Verlag, Denmark (1999)

29. Schach, S.R. Object-Oriented and Classical Software Engineering, $\mathbf{5}^{\text {th }}$ ed. McGraw-Hill, New York (2002)

30. Booch, G. Object-Oriented Analysis and Design with Applications. Addison-Wesley Publishing Company, California (1994)

31. Lucas, P. A Theory of Medical Diagnosis as Hypothesis Refinement. $6^{\text {th }}$ Conference on Artificial Intelligence, Springer-Verlag, New York (1997) 169-180 